Journal of trends in Computer Science and Smart technology (TCSST) (2020)

Vol.02/ No. 01

Pages: $37-45$

https://www.irojournals.com/tcsst/

DOI: https://doi.org/10.36548/jtcsst.2020.1.004

\title{
Sensor Assisted Incident Alarm System for Smart City Applications
}

\author{
Dr. Subarna Shakya \\ Professor, Department of Electronics and Computer Engineering, \\ Central Campus, Institute of Engineering, Pulchowk, \\ Tribhuvan University, \\ Pulchowk, Lalitpur Nepal. \\ Email: drss@ioe.edu.np.
}

\begin{abstract}
The advancement and development of proficient technologies that exploit sensors and things connected with the internet respectively along with the progress of the associated protocols has greatly encouraged the growth of applications that are basically dependent on the sensors for observing the happenings. Multitudes of such applications have been designed to have an improved discernment of the environment and sort out the exigent circumstances. The prevailing internet of things developed for identifying the exigent situations have restrictions when a wide space of smart cities are taken into consideration, as it has to employ many monitoring variables or even over burden the regular sensor that are engaged in monitoring. So the proposed method in the paper aims in developing a three-layered exigent incident alarm system utilizing the sensors considering the various data's gathered from the sensors and the geo-positioning system as input. The system modelled identifies and indicate the exigent situations using an alarm based on the incidents recognized and as well as the prior knowledge of the affected areas hazard's. By this the modularized and flexible awareness of the emergency events are delivered as per the details obtained from the smart city scenario. The evaluation of the proposed model proved its potentiality in assisting the progress in the creating awareness on the exigent situations using the internet of things.
\end{abstract}

Keywords: Internet of Things, Sensors, Incident Identification, Alarm System, Smart City Applications.

\section{Introduction}

The emergence of more effective and low-priced machineries for distributed data processing has concreted the way for innovative applications, allowing use of the IoT-centered machine-to-machine paradigm. The internet of things enabled to alleviate few common problems of the urban areas, causing developments in the cities. Recently multitudes of applications have been developed to observe the activities and the atmospheric changes that are taking place in the cities, delivering, the particulars about the events of the people, vehicles, buildings, animals, weather based on the information's acquired from the sensors. A large

ISSN: 2582-4104 
Journal of trends in Computer Science and Smart technology (TCSST) (2020)

Vol.02/ No. 01

Pages: $37-45$

https://www.irojournals.com/tcsst/

DOI: https://doi.org/10.36548/jtcsst.2020.1.004

amount of information's are gathered from the urban areas by engaging the sensor nodes. So the sensors engaged allows one to have the details of the unpredictable disaster that requires immediate response as it causes major economic losses. Hence sorting out an effective ways to regulate the exigent situations in these context are significant.

The sensor technology exploited to create awareness among the people on the disaster situations emphasis only on a particular phenomenon for instance either the flood, fire, rainstorm etc. lacking flexibility or adaptability in observing various scenarios. So this makes one to employ various number of essential system to alert the public or over burden the sensors engaged. So monitoring and reporting an exigent circumstances requires enhancement looking after the progress of such applications.

The exigent situations may be categorized based on its severity, and the damages it has created on the affected area as well as its time-based consequence, however the situations is it is necessary to track the direct line between an emergency as will the instance the events has occurred so as to turn on the alarm system. Although multitudes of ways exist to identify the events and the proposed method utilizes the Coordinates of the Geo-positioning system to identify the criticality of the incident occurred in a particular time instant followed by activating the alarm system. But the extent of the occurrence totally depends on the various installations that are adapted. For instance the detecting a smoke emission could be indicated by the temperature sensor but the intensity of the smoke level is required to activate the alarm system and the level of the alarms activated has to be varied depending on the population in the affected area.

So the proposed model in the paper devises an exceedingly configurable, more adaptable and effective exigent-incident alarm frame work that is three layered holding the applications, happenings and the incident alarms in each of its layers. The proposed method engages devices to identify (IU's) the occurrence of the events along with the location and the time instance of occurrence using the geo-positioning system, the computing units to process the information's gathered and the user applications registered.

The paper providing an incorporated exigent incident alarm model is framed with the related works disclosed in section two, the proposed system model in section three the performance evaluation in the section four and the Conclusion in section five.

ISSN: 2582-4104 
Journal of trends in Computer Science and Smart technology (TCSST) (2020)

Vol.02/ No. 01

Pages: $37-45$

https://www.irojournals.com/tcsst/

DOI: https://doi.org/10.36548/jtcsst.2020.1.004

\section{Related Works}

Su et al [1], has elaborated the details of the internet of things applications that has are necessitated in making a city smart Zygiaris, et al [2] has put forth the "Assisting planners to conceptualize the building of smart city innovation ecosystems" Valanarasu et al [3], has proposed the "Smart and Secure Iot and AI Integration Framework for Hospital Environment" Song, et al [4], discloses the "foundations and the principle required in developing a smart city"

Duraipandian et al [5] has put forth a "Cloud based Internet of Things for smart connected objects." Reyes, et al [6] has developed an "Computer-enabled, networked, facility emergency notification, management and alarm system." Tewolde et al [7] has put forth the "Sensor and network technology for intelligent transportation systems." In the development of smart cities.

Gharaibeh et al[8] elaborates the "A survey on data management, security, and enabling technologies for the smart cities" Smys, S., et al [9] discusses the "Introduction to the Special Section on Inventive Systems and Smart Cities." Bhatti et al [10] has put forth the "A novel internet of things-enabled accident detection and reporting system for smart city environments."

Alharbi, N., et al [11] has developed a "the challenges, involvement and the activities of the sensors in the smart cities" Jennifer S. Raj et al [12] has put forth the "Internet of Things and Big Data Analytics for Health Care with Cloud Computing." More et al [13] proposes the "Smart Goods Transportation system using Internet of Vehicle." Ashwin, Dr M. et al [14] has devised a solution for the challenges faced in the application of smart cities.

Bhatt et al [15] "disclosed the methodology to devise a traffic sign board that is smart for the smart cities" J. Vijitha Ananthi et al [16] proposed an IoT based " automated greenhouse environment" that monitors the changes in temperature, humidity and the pressure and Stoyanov, et al constructed a novel reference structure that supports the applications of the smart city.

ISSN: 2582-4104 
Journal of trends in Computer Science and Smart technology (TCSST) (2020)

Vol.02/ No. 01

Pages: $37-45$

https://www.irojournals.com/tcsst/

DOI: https://doi.org/10.36548/jtcsst.2020.1.004

\section{Proposed Incident Alarm Frame work}

This three layered incident alarm frame work is organized with the layers representing the registered user application, the processor units to processing the severity of the events gathered and the identification unit to detect the occurrence of the events with the help of the sensors and the geo-positioning units to locate the instance and the position of the incident occurrences. The figure. 1 below is the proposed model of the three layered incident detection system.

\section{Identification unit}

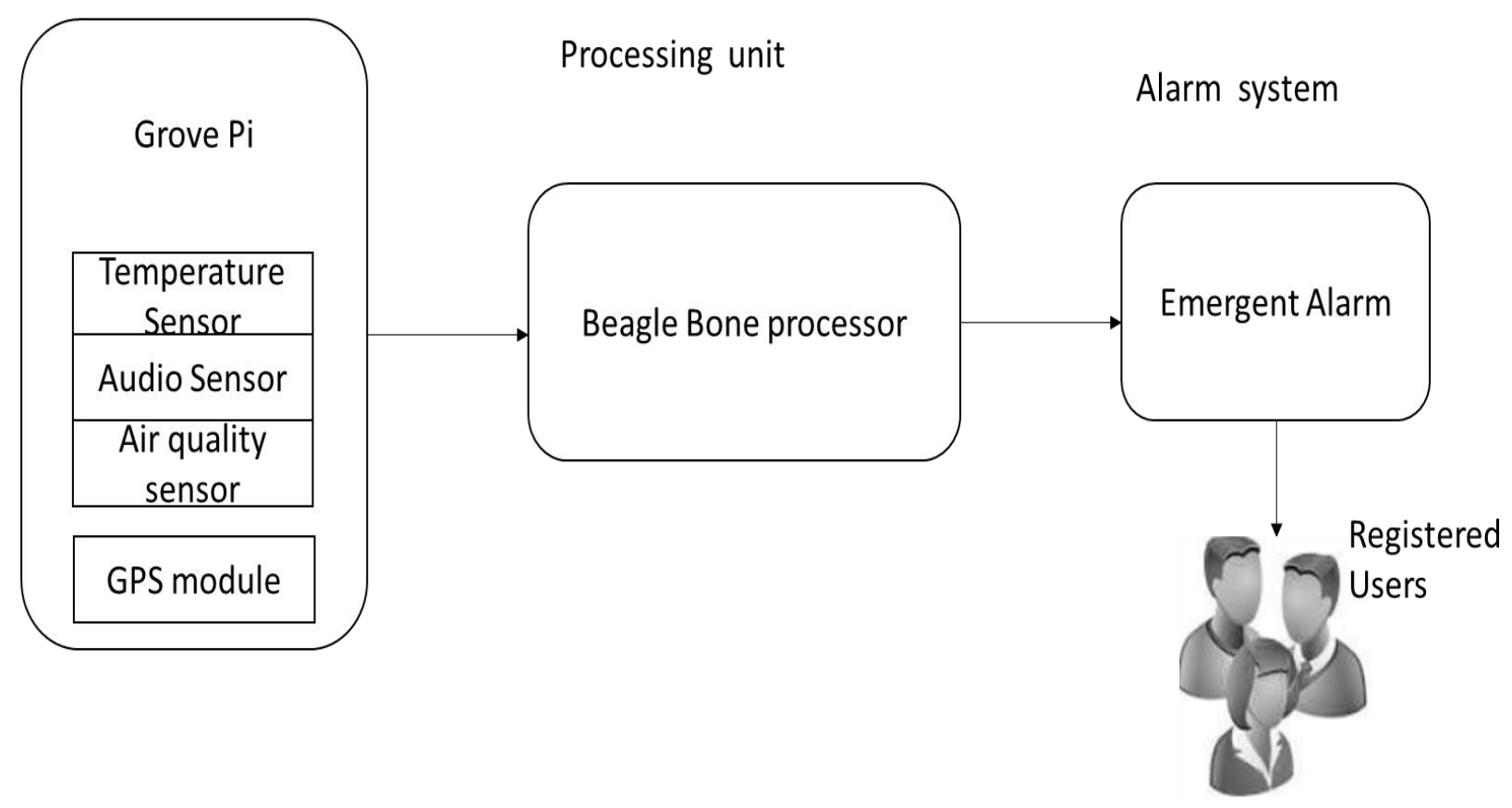

Figure.1 Proposed Block Diagram

To enhance the flow of communication the proposed model was devised using the following in each of its layers. The identification unit which is the very basic block of the system to raise alarm is encompassed with grove of sensors to identify the incidents, this layer is also equipped with the geo positioning system

ISSN: 2582-4104 
Journal of trends in Computer Science and Smart technology (TCSST) (2020)

Vol.02/ No. 01

Pages: $37-45$

https://www.irojournals.com/tcsst/

DOI: https://doi.org/10.36548/jtcsst.2020.1.004

to give information's regarding the position of occurred incidents. The next layer holds the processor, the proposed model uses the beagle-bone black processors [5] to process the sensed data's accumulated based on the manually defined information's (the magnitude, the geographical scope, temporal importance and the occurred duration) that are organized according to the city taken into consideration and the time when the incident occurred. With the help of the processor the exigent alarms are triggered on the emergent situations. The identification units are aware of the processors positions, and number of processors engaged vary depending on the size of the city. The final layer is equipped with the incident emergent alarm that is communicated by the processing system. These emergent alarms are triggered only for the user applications that have registered with the processor utilizing the basic registration process, the emergent alarm is represented using the JSON format delivering the its particulars such as identification number along with the level of the severity. The flow chart below in figure. 2 describes the steps in event identification and processing

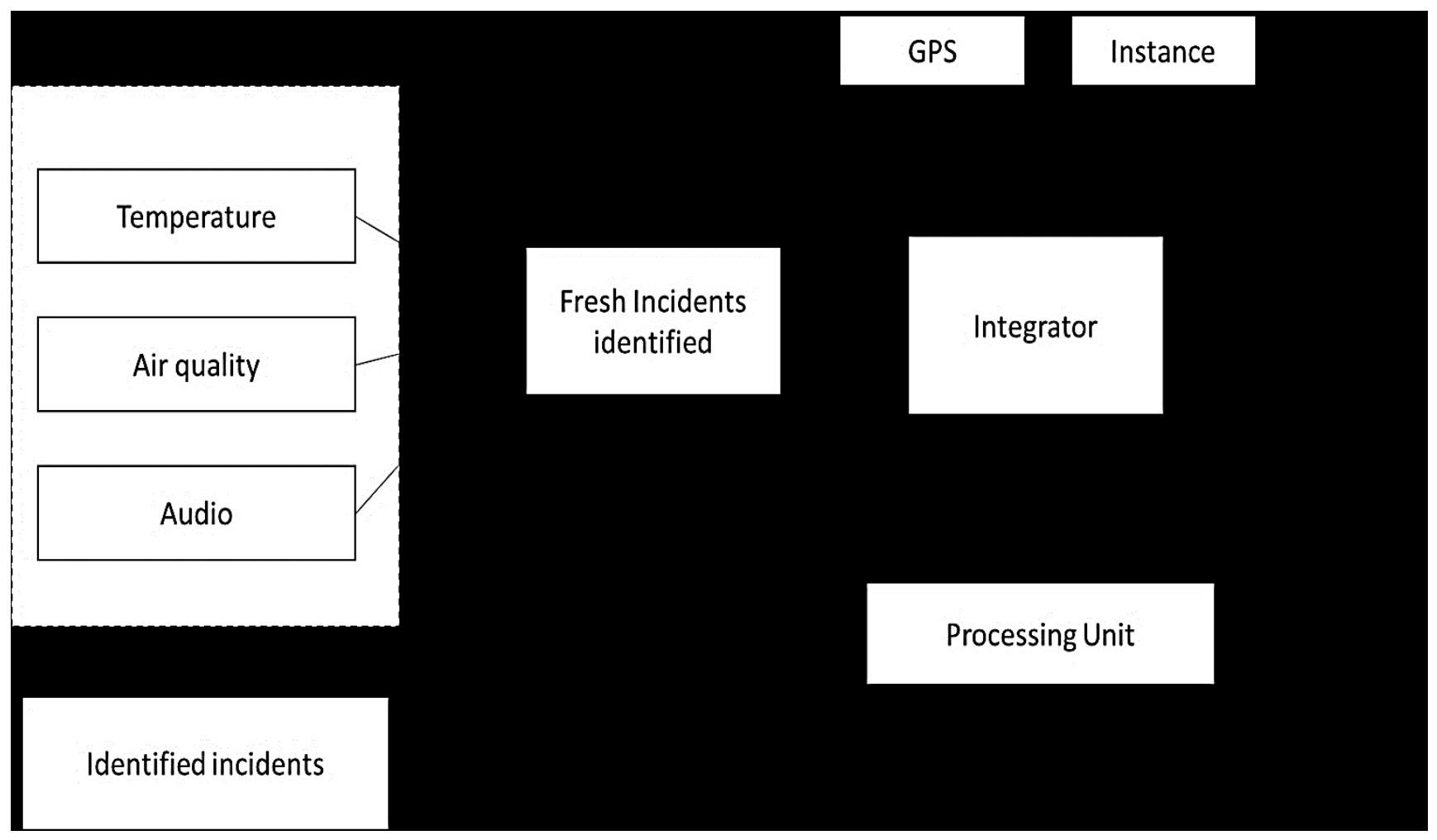

ISSN: 2582-4104 
Journal of trends in Computer Science and Smart technology (TCSST) (2020)

Vol.02/ No. 01

Pages: $37-45$

https://www.irojournals.com/tcsst/

DOI: https://doi.org/10.36548/jtcsst.2020.1.004

Figure.2 The identification and the Processing Unit

As shown in the figure.2, the data's are transmitted only on detecting the incidents and system remains idle if no incidents are detected. On identifying even one incident the data's are transmitted to the Processing units. During the frequency set for sensing the identified reports are conveyed to the unit that regulates the information flow. The figure. 3 below shows the "communication flow in the proposed system that is enabled by the message, queuing protocol"

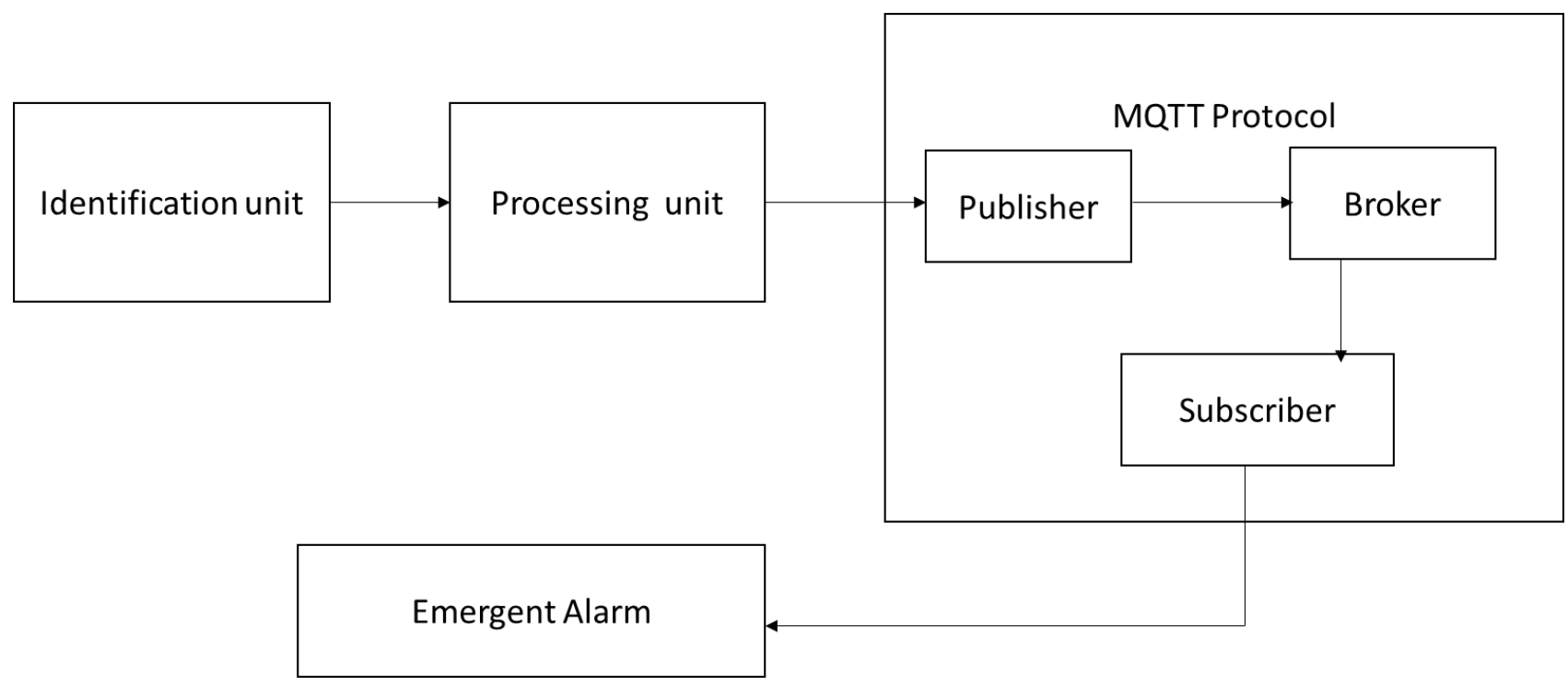

Figure.3 "Data Communication using MQTT Protocol”

The identification unit is installed engaging more than one sensors, allotting an identification tag for each sensor, the frequency for sensing and refreshing are set, the frequency of refreshing $=5 *$ frequency of sensing. The verge for each incident identification is set, the data are sensed based on the different types of sensors engaged. The processing units TCP and the IP address are fed to the identification unit and the coding is done to feed the details of TCP and the IP address into the identification units. The processing unit's waits for the fresh request, the fresh request are processed under "separate threads" The unit fixed for processing is basically a "software implementation" that could be executed even using a PC. The coordinates of the geo positioning unit are defined into the unit that performs processing to gain knowledge on the position of the incident occurrence. The MQTT protocol is utilized to extend the communication

ISSN: 2582-4104 
Journal of trends in Computer Science and Smart technology (TCSST) (2020)

Vol.02/ No. 01

Pages: $37-45$

https://www.irojournals.com/tcsst/

DOI: https://doi.org/10.36548/jtcsst.2020.1.004

across the unit that computes the data and the emergent alarm. "The MQTT Broker is the Mosquitto (https:/mosquitto.org), a popular open-source MQTT broker that has sponsored several IoT and Smart City initiatives. In the experiments performed, the Mosquitto Broker was implemented on an out-and-out beagle bone black board and disclosed in the Alarm units-Mosquitto broker, the MQTT messages are managed using the Python paho-mqtt library" the coding for the module present in the each layer is written using the python and their enactments are ordered in the corresponding directories.

\section{Performance evaluation}

In preceding parts, the proffered emergent alarm system was thoroughly defined, defining the characteristics and function of the elements relevant to the identification of incidents and broadcasting of emergent-alarms in the urban areas. Nevertheless, due the meticulousness, of the proffered system assessments with former systems might be insignificant, it is a versatile multi-event distributed emergency warning system with some revolutionary ideas, such as how incidents are perceived and the severity level are identified. So the model put forth in the paper is evaluated on the current smart city with emergent situations. The table. 1 below shows the percentage of monitoring possibility, incident detection, scalability and difficulties in configuring.

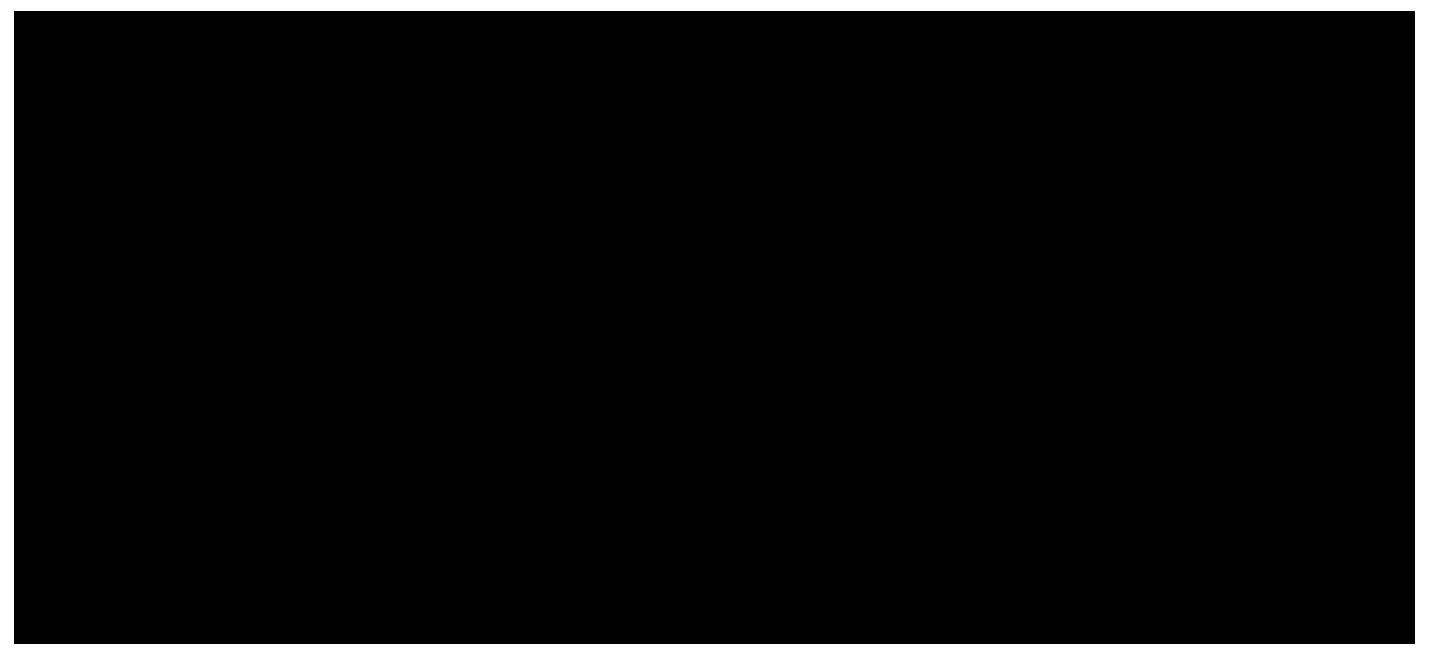

Table.1 Percentage of Monitoring Possibility, Incident Detection, Scalability and Difficulties in Configuring. 
Journal of trends in Computer Science and Smart technology (TCSST) (2020)

Vol.02/ No. 01

Pages: $37-45$

https://www.irojournals.com/tcsst/

DOI: https://doi.org/10.36548/jtcsst.2020.1.004

It was found the emergent alarm put forth in the paper was capable of handling any type of exigent circumstance, eluding the suitability and the interoperability problems that would take place when more than one systems are uninterruptedly functioning. It is further more adept in identifying the emergent circumstance as it detects the incidents based on the number of events, hazardousness area and its historical significance. More over the systems scalability was very much better as it flexible, adaptable and skillful in detecting simultaneously more than one incident. The difficulties in configuring the system was found to be very low as the proposed as stated previously was highly adaptive to any system and various levels of monitoring allowing the computations to be performed even by other systems.

\section{Conclusion}

The paper puts forth the novel model to indicate the emergent situations in the smart city, exploiting the sensors and the alarm system, the system is basically devised using the three layers equipped with the sensors, processors and the alarm system respectively, the alarm is raised only on detecting emergent circumstance only to the user application registered with it. The complete process was implemented using the python, and evaluated on different cities with exigent scenarios on the basis of the monitoring possibility, incident detection, scalability and difficulties in configuring. It was found the proposed model had an effective monitoring and delivered better identification of incidents and broadcasting of emergentalarms in the urban areas. Its meticulousness made the comparative assessment of the proposed model with the former monitoring system insignificant.

\section{References}

[1] Su, Kehua, Jie Li, and Hongbo Fu. "Smart city and the applications." In 2011 international conference on electronics, communications and control (ICECC), pp. 1028-1031. IEEE, 2011.

[2] Zygiaris, Sotiris. "Smart city reference model: Assisting planners to conceptualize the building of smart city innovation ecosystems." Journal of the knowledge economy 4, no. 2 (2013): 217231.

[3] Valanarasu, Mr R. "Smart and Secure Iot and AI Integration Framework for Hospital Environment." Journal of ISMAC 1, no. 03 (2019): 172-179.

[4] Song, Houbing, Ravi Srinivasan, Tamim Sookoor, and Sabina Jeschke. Smart cities: foundations, principles, and applications. John Wiley \& Sons, 2017.

[5] Duraipandian, M., and R. Vinothkanna. "Cloud based Internet of Things for smart connected objects." J. ISMAC 1, no. 02 (2019): 111-119.

ISSN: 2582-4104 
Journal of trends in Computer Science and Smart technology (TCSST) (2020)

Vol.02/ No. 01

Pages: $37-45$

https://www.irojournals.com/tcsst/

DOI: https://doi.org/10.36548/jtcsst.2020.1.004

[6] Reyes, Tommy Dean, and Garry Oren Thompson. "Computer-enabled, networked, facility emergency notification, management and alarm system." U.S. Patent 7,277,018, issued October 2, 2007.

[7] Tewolde, Girma S. "Sensor and network technology for intelligent transportation systems." In 2012 IEEE International Conference on Electro/Information Technology, pp. 1-7. IEEE, 2012.

[8] Gharaibeh, Ammar, Mohammad A. Salahuddin, Sayed Jahed Hussini, Abdallah Khreishah, Issa Khalil, Mohsen Guizani, and Ala Al-Fuqaha. "Smart cities: A survey on data management, security, and enabling technologies." IEEE Communications Surveys \& Tutorials 19, no. 4 (2017): 2456-2501.

[9] Smys, S., Hui-Ming Wee, and Meng Joo. "Introduction to the Special Section on Inventive Systems and Smart Cities." (2018): 32-33.

[10] Bhatti, Fizzah, Munam Ali Shah, Carsten Maple, and Saif Ul Islam. "A novel internet of thingsenabled accident detection and reporting system for smart city environments." Sensors 19, no. 9 (2019): 2071.

[11] Alharbi, N., and B. Soh. "Roles and Challenges of Network Sensors in Smart Cities." In IOP Conference Series: Earth and Environmental Science, vol. 322, no. 1, p. 012002. IOP Publishing, 2019.

[12] Smys, S., and Jennifer S. Raj. "Internet of Things and Big Data Analytics for Health Care with Cloud Computing." Journal of Information Technology 1, no. 01 (2019): 9-18.

[13] More, Sunanda, Shraddha Pawar, and Lalita Gavit. "Smart Goods Transportation system using Internet of Vehicle." (2019).

[14] Ashwin, Dr M., Bhavani Vasantha, A. Roshini, Dr Anguraj, and Dinesh Kumar. "Smart City Applications with Solutions-A Survey." International Journal of Psychosocial Rehabilitation 23, no. 4 (2019).

[15] Bhatt, Devershi Pallavi, and Manish Tiwari. "Smart Traffic Sign Boards (STSB) for Smart Cities." (2019): 21-4.

[16] Raj, Jennifer S., and J. Vijitha Ananthi. "Automation Using Iot in Greenhouse Environment." Journal of Information Technology 1, no. 01 (2019): 38-47.

[17] Stoyanov, Stanimir, Todorka Glushkova, Asya Stoyanova-Doycheva, and Vanya Ivanova. "A Reference Architecture Supporting Smart City Applications." In International Conference on Business Information Systems, pp. 463-474. Springer, Cham, 2019.

ISSN: 2582-4104 